\title{
Artérias mesentéricas cranial e caudal em mocós Kerodon rupestris (Wied, 1820) ${ }^{1}$
}

\author{
Paula V.S. Queiroz ${ }^{2}$, Carlos E.B. Moura ${ }^{3}$, Jesane A. Lucena ${ }^{4}$, Moacir F. Oliveira ${ }^{4}$ \\ e José F.G. Albuquerque ${ }^{4}$
}

\begin{abstract}
Queiroz P.V.S. Moura C.E.B., Lucena J.A., Oliveira, M.F. \& Albuquerque J.F.G. 2011. [Cranial and caudal mesenteric arteries in rock cavy Kerodon rupestris (Wied, 1820).] Artérias mesentéricas cranial e caudal em mocós Kerodon rupestris (Wied, 1820). Pesquisa Veterinária Brasileira 31(7):623-626. Departamento Ciência Animal, Universidade Federal Rural do Semi-Árido, Bairro Presidente Costa e Silva, Mossoró, RN 59625-900, Brazil. E-mail: moacir@ufersa.edu.br

In this study about the origin and ramification of the cranial (CrMA) and caudal (CaMA) mesenteric collateral arteries of the rock cavy, 20 animals (18 males and 2 females) of different ages, originated from the Wild Animals Multiplication Center of the Universidade Federal Rural do Semi-Árido (Cemas/Ufersa), were used. After the natural death, the walls of the abdominal cavity of the animals, in the left antimere, were dissected to cannulate to the aorta in prediaphragmatic path. Then they were fixed in $10 \%$ formaline and conditioned in order to study their anatomy. The results showed that in 18 animals (90\%) the CrMA arose, separately, of the abdominal aorta, soon after the celiac artery (CA), originating, by this time, the middle colic (MCo), caudal pancreaticduodenal (CPD), duodenojejune (DJ), jejune (J) and ileocecocolic (ICeCo) trunk from which derives the ileocecal (ICe) and the right colic (RCo) arteries. In one rock cavy (5\%), the CrMA and CA originate from abdominal aorta in a common trunk. In this case the CrMA originated the CPD, MCo, ICeCo, and J. In one observation (5\%) CrMA and CaMA appear in common trunk. In this animal, CPD, DJ, ICeCo, MCo and J arteries were originated of the CrMA, while the left colic (LCo) and rectal cranial (RCr) arteries were originated of the CaMA. Regarding the CaMA, in 20 cases $(100,00 \%)$ it originates the LCo and the rectal cranial arteries.
\end{abstract}

INDEX TERMS: Irrigation, wild rodents, anatomy, abdominal and pelvic organs.

RESUMO.- No estudo sobre a origem e ramificações das artérias mesentéricas cranial (AMCr) e caudal (AMCa) do mocó, foram utilizados 20 animais (18 machos e 2 fêmeas) de diferentes idades, que, após morte natural, foram dissecados rebatendo-se as paredes torácica e abdominal, pelo antímero esquerdo, expondo-se a aorta que foi então canulada em seu trajeto pré-diafragmático, procedendo-se a injeção de neoprene látex corado, no sentido caudal. A seguir, foram fixados

${ }^{1}$ Recebido em 9 de setembro de 2010.

Aceito para publicação em 4 de março de 2011.

Departamento de Enfermagem, Universidade do Estado do Rio Grande do Norte (UERN), Rua André Sales s/n, Bairro Paulo VI, Caicó, RN 59300-000, Brasil. paulavivianne@yahoo.com

${ }^{3}$ Departamento de Morfologia, Universidade Federal do Rio Grande do Norte (UFRN), Caixa Postal 1524, Av. Salgado Filho s/n, Campus Universitário Lagoa Nova, Natal, RN 59072-970, Brasil.

${ }^{4}$ Departamento Ciências Animais, Universidade Federal Rural do SemiÁrido (Ufersa), BR $110 \mathrm{Km} \mathrm{47,} \mathrm{Bairro} \mathrm{Pres.} \mathrm{Costa} \mathrm{e} \mathrm{Silva,} \mathrm{Mossoró,} \mathrm{RN}$ 59625-900, Brasil. *Autor para correspondência: moacir@ufersa.edu.br em solução aquosa de formol a 10\%, durante 48 horas, e posteriormente dissecados. Os resultados mostraram que em 18 animais (90\%), a AMCr originou-se da aorta abdominal isoladamente, logo após a artéria celíaca, emitindo as artérias cólica média ( $\mathrm{CoM})$, pancreaticoduodenal caudal (PDC), duodenojejunal (DJ), jejunal (J) e ileocecocólica (ICeCo). Em um mocó (5\%), as AMCr e AC se originaram da aorta abdominal em um tronco comum. Neste caso, a $\mathrm{AMCr}$ originou às artérias CoM, PDC, ICeCo e J. Em uma observação (5\%), as artérias $\mathrm{AMCr}$ e AMCa surgiram em tronco comum. Neste animal, as artérias PDC, DJ, ICeCo, CoM e J foram originadas da $\mathrm{AMCr}$, enquanto as aterias cólica esquerda $(\mathrm{CoE})$ e retal cranial (ARCr) derivaram da AMCa. Dois animais (10\%) apresentaram como colaterais da $\mathrm{AMCr}$ as artérias CoM, PDC, DJ, $\mathrm{J}$ e o tronco ICeCo, que originou às artérias $\mathrm{CoD}$ e ileocecal (ICe). No que diz respeito a AMCa, nos 20 casos (100\%) originou as artérias CoE e RCr.

TERMOS DE INDEXAÇÃO: Irrigação, roedores silvestres, anatomia, órgãos abdominais e pélvicos. 


\section{INTRODUÇÃO}

Rodentia é a maior ordem dentre os Eutheria. Os roedores são animais que apresentam ampla tolerância ecológica, habitando ambientes com condições variadas de climas e altitudes. Essas características comportamentais são refletidas diretamente nas adaptações funcionais do organismo desses animais.

Os mocós são representantes da subordem Hystrichomorpha, que se caracterizam por apresentarem a superfície dorsal do corpo com coloração cinza-clara, mesclada de preto e branco e a parte posterior das coxas de coloração castanho-ferriginosa (Moojen 1952). Quando a adultos medem até 410 milímetros e podem apresentar até um quilograma de peso vivo. São desprovidos de clavículas e têm mandíbula alongada, na qual os dentes se distribuem de maneira especialmente particular quando comparado a outros tipos de roedores. Possuem cauda e pés tridáctilos, os quais são dotados de coxins calosos e unhas rígidas, o que lhes fornecem extrema habilidade para galgar superfícies pedregosas e troncos de árvores. Acerca de sua morfologia são poucos os trabalhos encontrados na literatura, possivelmente, pelo fato deste grupo de animais está restrito a região Nordeste e ao norte do estado de Minas gerais. Contudo são descritos trabalhos desenvolvidos por Magalhaes et al. (2008), Lima et al. (2008), Oliveira et al. (2006), Thomaz et al. (2006), Lacerda et al. (2006), Zogno et al. (2004) e Oliveira et al. (2003). Na literatura não se tem conhecimento acerca do arranjo das artérias mesentéricas nesse animal. Contudo, existem diversas referências ao estudo desses vasos em outras espécies silvestres.

Albuquerque et al. (1976) descreveram a ocorrência de um tronco comum das artérias celíaca e mesentérica cranial em saguis (Callithrix jacchus), comportamento observado também em macacos-prego (Cebus nigritus) por Borelli et al. (1971) e em gambás (Didelphis aurita) por Albuquerque et al. (1975).

Albuquerque et al. (1976) notaram em Callithrix jacchus a ocorrência da artéria cólica média e do tronco de origem das artérias jejunal e ileocecocólica.

Em gambás (Didelphis aurita), Figueiredo et al. (1979) observaram a ocorrência das artérias cólica média, jejunal, ileocecocólica como ramos oriundos da artéria mesentérica cranial. Silva et al. (1996) descrevem a ocorrência das artérias pancreaticoduodenal caudal, cólica média, jejunal e ileocecocólica em Cebus apella.

Os troncos provenientes da artéria mesentérica cranial em veado catingueiro (Mazama govazoubira) foram descritos por Souza et al. (1996) como sendo as artérias pancreática, cólica média, ileocecocólica, jejunal e ileal.

Atalar et al. (2005), em um estudo com a artéria mesentérica cranial em porco-espinho (Hystrix cristata) observaram que esta emite como ramos as artérias cólica média, cólica direita, pancreaticoduodenal caudal e os troncos jejunal e ileocecocólico.

0 presente estudo tem por objetivo descrever o comportamento das artérias mesentéricas e seus ramos no Kerodon rupestris, dada a importância desses vasos no suprimento sanguíneo de diversos órgãos abdominais. A compreensão da fisiologia desses animais através de análises morfo-funcionais é de extrema importância para a tomada de medidas que favoreçam sua preservação.

\section{MATERIAL E MÉTODOS}

Foram estudados 20 mocós (18 machos e 2 fêmeas) de diferentes idades, provenientes do Centro de Multiplicação de Animais Silvestres, cativeiro com fins científicos autorizado pelo IBAMA através do $\mathrm{n}$ - 1478912, que por ocasião de morte natural foram doados ao Laboratório de Anatomia Veterinária da Universidade Federal Rural do Semi-Árido (UFERSA).

Os animais foram dissecados rebatendo-se as paredes torácicas e abdominal, pelo antímero esquerdo, expondo-se a aorta que foi então canulada em seu trajeto pré-diafragmático, procedendo-se a injeção de neoprene látex, devidamente corado, no sentido caudal. A seguir, foram fixados em solução aquosa de formol a 10\%, durante 48 horas, e posteriormente dissecados.

De todas as observações foram feitos esquemas gráficos que serviram de base para as análises e documentação.

Os termos anatômicos utilizados nas descrições estão de acordo com International Committee on Veterinary Gross Anatomical Nomenclature, 2005.

\section{RESULTADOS}

Em mocós, as artérias mesentéricas cranial e caudal originaram-se da aorta abdominal, ora isoladas ( $95 \%$ dos casos), ora em tronco comum (5\%). Neste trabalho verificou-se que em $50 \%$ dos casos (10 animais), a artéria mesentérica cranial, depois de surgir isoladamente da aorta abdominal, forneceu na seguinte ordem as artérias: cólica média, pancreaticoduodenal caudal, duodenojejunal, jejunal e o tronco ileocecocólico. Nestes animais, o tronco ileocecocólico representou uma continuação da artéria mesentérica cranial e dele derivaram às artérias ileocecal e cólica direita (Fig.1A). Em 25\% da amostra (5 animais), a artéria mesentérica cranial originou os mesmo ramos supracitados, entretanto o tronco ileocecocólico surgiu, após as artérias cólica média e pancreaticoduodenal caudal, como terceiro ramo. Nestes animais, a continuação da artéria mesentérica cranial originou um tronco do qual surgiam as artérias jejunais. (Fig.1B).

Em 10\% dos casos (2 animais), da artéria mesentérica cranial surgiu como primeiro ramo a artéria pancreaticoduodenal caudal, seguida de dois ramos terminais, um correspondia ao tronco ileocecocólico e do outro derivaram as artérias duodenojejunal, jejunais e cólica média (Fig.1C).

Em um animal (5\%) observou-se que na ausência do tronco ileocecocólico, as artérias ileocecal e cólica direita surgiam diretamente da artéria mesentérica cranial. Neste caso, a artéria pancreaticoduodenal caudal, também figurou como primeiro ramo da mesentérica cranial (Fig.1D).

Em um mocó (5\%) foi observado a ocorrência do tronco celíacomesentérico surgindo da aorta abdominal, neste caso, a artéria mesentérica cranial forneceu as artérias pancreaticoduodenal caudal, cólica média, ileocecocólica e jejunal e da artéria celíaca surgiram as artérias hepática, gástrica esquerda e lienal (Fig.1E).

Em um animal (5\%) observou-se uma variação incomum na origem das artérias mesentéricas cranial e caudal. Esses dois vasos surgiam de um tronco derivado da aorta abdominal. Em seguida, artéria mesentérica originou as artérias pancreaticoduodenal caudal, duodenojejunal, ileocecocólica, cólica média e jejunal. Enquanto, a artéria mesentérica caudal ramificou-se nas artérias cólica esquerda e retal cranial (Fig.1F). No entanto, em 95\% dos casos (19 animais), a arté- 


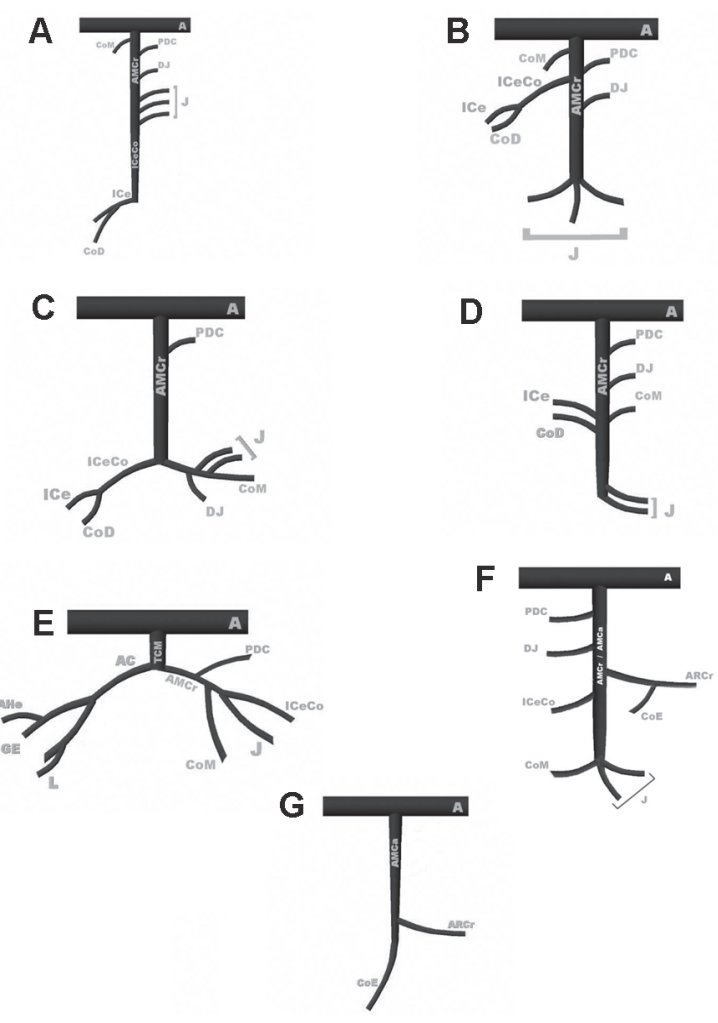

Fig.1. Disposição dos colaterais das artérias mesentéricas cranial e caudal em mocós. (A) Aorta abdominal, (AC) Artéria celíaca, (AHe) Artéria hepática, (AMCa) Artéria mesentérica caudal, (AMCr) Artéria mesentérica cranial, (ARCr) Artéria retal cranial, (CoD) cólica direita, (CoE) cólica esquerda, (CoM) cólica média, (DJ) duodenojejunal, (GE) gástrica esquerda, (Ice) ileocecal, (ICeCo) ileocecocólica, (ICo) ileocólica, (J) jejunal, (L) lienal, (PDC) pancreaticoduodenal caudal, (TCM) Tronco celiacomesenterico.

ria mesentérica caudal surgiu como ramo isolado da aorta abdominal, e em seguida, também, dividiu-se nas artérias cólica esquerda e retal cranial (Fig.1G).

\section{DISCUSSÃO}

As artérias mesentéricas cranial e caudal são consideradas ramos viscerais da aorta abdominal, conforme classificação dos colaterais da aorta descendente proposta por Dyce et al. (2004), sendo responsáveis pela irrigação dos intestinos (intestino caudal). Sua origem, ramificação e distribuição tem sido objeto de estudo de várias pesquisas envolvendo animais silvestres. Apesar de este ser o primeiro trabalho que aborda aspectos atinentes à ramificação das artérias mesentéricas cranial e caudal em Kerodon rupestris, Nossos resultados permitem uma comparação com aqueles verificados em animais domésticos e espécies silvestres já estudadas, especialmente roedores.

Em 90\% dos mocós estudados, a artéria mesentérica cranial originou-se como ramo isolado da aorta abdominal logo após a origem da artéria celíaca, resultados semelhantes foram descritos por Machado et al. (2005) em 70\% (7 animais) dos ratões-do-banhado analisados e por Souza et al. (1996) em $50 \%$ da amostra de veados catingueiros estudada, bem como em $100 \%$ dos coelhos da raça Nova Zelândia estudados por Abidu-Figueiredo et al. (2008).
Em 5\% da amostra, foi observado um tronco celiacomesentérico, esta variação anatômica foi relatada em diversas espécies, 39,3\% dos ovinos por Langenfeld \& Pastea (1977); em 9,67\% dos bubalinos por Machado et al. (2000); em 6,66\% dos caprinos por Ferreira et al. (2001); em 4,7\% dos felinos por Peduti Neto \& Borelli (1970) e em um cão por Schmidt \& Schoenau (2007). Esse comportamento também foi observado por Albuquerque et al. (1976) em 30\% dos sagüis e por Machado et al. (2006) em 30\% dos ratões-do-banhado. 0 referido tronco também foi descrito por Borelli et al. (1971) em Cebus nigritus e por Silva et al. (1996) com Cebus apella.

Em 75\% dos mocós analisados a artéria cólica média apareceu como o primeiro ramo originado da artéria mesentérica cranial. Estes achados são semelhantes aos descritos por Atalar et al. (2005) para 78\% dos roedores Hystrix cristata estudados e por Machado et al. (2006) em 40\% dos ratõesdo-banhado analisados.

A artéria pancreaticoduodenal caudal havia sido descrita como primeiro ramo da artéria mesentérica cranial em 100\% dos coelhos da raça Nova Zelândia pesquisados por Machado et al. (2008) e 60\% dos ratões-do-banhado estudados por Machado et al. (2006). Em mocós, esse comportamento ocorreu em $25 \%$ da amostra (5 animais).

A artéria duodenojejunal esteve presente em 95\% dos mocós estudados, com origem diretamente da artéria mesentérica cranial, imediatamente após as artérias cólica média e pancreaticoduodenal caudal, comportamento semelhante havia sido descrito em $90 \%$ dos ratões-do-banhado estudados por Machado et al. (2006).

O tronco ileocecocólico representou uma continuação da artéria mesentérica cranial em 50\% dos mocós analisados. Atalar et al, (2005) já havia observado esse arranjo em Hystrix cristata, Nickel et al. (1981) afirmaram ser este comportamento comum em roedores. Esse tronco é descrito, também, por Figueiredo et al. (1979), em gambás, Silva et al. 1996), em macaco-prego, Albuquerque et al. (1976) em sagui e ainda por Souza et al. (1996) no veado catingueiro. No mocó, observou-se que deste tronco originou-se as artérias ileocecal e cólica direita, no enquanto em ratão-do-banhado (Machado et al. 2006) e no Hystrix cristata (Atalar et al, 2005), a artéria cólica direita surgiu diretamente da artéria mesentérica cranial e fazia anastomose com o ramo cólico da artéria ileocólica.

Em 100\% dos mocós, a artéria mesentérica caudal emitiu como ramos as artérias cólica esquerda e retal cranial, comportamento semelhante foi descrito em sagüis por Albuquerque et al. (1976), em gambás por Figueiredo et al. (1979), em veados catingueiros por Souza et al. (1996) , em cutias por Carvalho et al. (1999), em macacos-prego por Silva et al. (1996) e em ratão-do-banhado por Machado et al. (2006). A frequencia com que a artéria mesentérica caudal origina as artérias cólica esquerda e retal cranial, nas diferentes espécies, parece indicar um padrão de vascularização para diferentes espécies silvestres.

O tronco comum dando origem as artérias mesentéricas cranial e caudal configura um comportamento incomum. Não existem referências a esse tipo de arranjo na literatura clássica da anatomia veterinária e muito menos na literatura especializada ao nosso alcance. 


\section{CONCLUSÕES}

No mocó, as artérias mesentéricas cranial e caudal apresentaram seis arranjos de origem e ramificações, sendo o mais frequente $(95 \%)$ aquele no qual se originavam isoladas da aorta abdominal.

A artéria mesentérica cranial emitiu, mais frequentemente, as seguintes artérias: cólica média, pancreaticoduodenal caudal, duodenojejunal, jejunal e ileocecocólica. Enquanto, da artéria mesentérica caudal derivou, em todos os casos, as artérias cólica esquerda e retal cranial.

\section{REFERÊNCIAS}

Abidu-Figueiredo M., Xavier-Silva B., Cardinot T.M., Babinski M.A. \& Chagas M.A. 2008. Celiac artery in New Zealand rabbit: Anatomical study of its origin and arragement for experimental research and surgical practice. Pesq. Vet. Bras. 28(5):237-240.

Albuquerque J.F.G., D’Errico A.A. \& Pedutti Neto J. 1975. Origem das artérias celíaca e mesentérica cranial, por tronco comum, em gambás (Didelphis aurita). Científica, Universidad del Zulia, Maracaibo, 3(1):39-41.

Albuquerque J.F.G., Fernandes Filho A. \& Borelli V. 1976. Contribuição ao estudo da ramificação das artérias mesentéricas cranial e caudal em sagüis (Callithrix jacchus). Revta Fac. Med. Vet. Zootec. USP, São Paulo, 13(1):61-65.

Atalar O. \& Yilmaz S. 2005. The cranial mesenteric artery in the porcupine (Hystrix cristata). Veterinarski Arhiv 75(1):75-82.

Borelli V., Pedutti Neto J. \& Santis Prada I.L. 1971 Origem das artérias celíaca e mesentérica cranial por tronco comum em macaco prego (Cebus nigritus). Revta Fac. Med. Vet. Zootec. USP, São Paulo, 8(3):539-581.

Carvalho M.A.M., Miglino M.A., Didio L.J.A. \& Melo A.P.F. 1999. Artérias mesentéricas cranial e caudal em cutias (Dasyprocta aguti). Veterinária Notícias 2:17-24.

Dyce K.M., Sack W.O. \& Wensing C.J. 2004. Tratado de Anatomia Veterinária. 3a ed. Editora Elsevier, Rio de Janeiro. 813p.

Ferreira F.A. Miglino, M.A., Silva F.O.C., Carvalho F.S.R. \& Santos T.C. 2001. Origem das artérias mesentéricas cranial e caudal em fetos de caprinos da raça Saanen (Capra hircus Linnaeus, 1758). Braz. J. Vet. Res. Anim. Sci. 38 (2):69-73.

Figueiredo S.F., Albuquerque J.F.G. \& Souza W.M. 1979. Contribuição ao estudo da ramificação das artérias mesentéricas cranial e caudal em gambás (Didelphis aurita). Científica, Universidad del Zulia, Maracaibo, Volume Especial:51-53.

International Committee on Veterinary Gross Anatomical Nomenclature 2005. Nomina Anatomica Veterinaria. $4^{\text {th }}$ ed. Word Association of Veterinary Anatomists, Ithaca, NY.

Lacerda P.M.O., Moura C.E.B., Miglino M.A., Oliveira M.F. \& Albuquerque J.F.G. 2006. Origem do plexo lombossacral de mocó (Kerondo rupestris). Braz. J. Vet Res. Anim. Sci. 43:620-628.
Langenfeld M. \& Pastea E. 1977. Anatomical variants of the celiac artery in sheep with special reference to the celiacomesenteric arterial trunk. Anatomischer Anzeiger 142:166-174.

Lima M.C., Bonattelli M., Oliveira M.F., Miglino M.A., Goiozo P.F.I., Massani S.M., Martins K. \& Carvalho A.F. 2008. Glândula mamária do mocó (Kerodon rupestris Weid Neuwied, 1820): aspectos morfológicos. Braz. J. Vet. Res. Anim. Sci. 45:88-93,

Machado M.R.F., Miglino M.A., Cabral V.P. \& Araújo N. 2000. Origem das artérias celíaca e mesentérica cranial em bubalinos (Bubalus bubalis L., 1758). Braz. J. Vet. Res. Anim. Sci, 37(2):99-104.

Machado G.V., Gonçalves P.R., Parizzi A. \& Souza J.R. 2006. Padrão de divisão e distribuição das artérias mesentéricas no ratão-do-banhado (Myocastor coypus, Rodentia: Mammalia). Biotemas 19(1):59-63.

Magalhães M.S., Albuquerque J.F.G., Oliveira M.F., Papa P.C. \& Moura C.E.B. 2007. Ramos do arco aórtico no mocó (Kerodon rupestris). Revta Port. Ciênc. Vet. 102(561/562):49-52.

Moojen J. 1952. Os Roedores do Brasil. Ministério de Educação e da Saúde. Instituto Nacional do Livro, Biblioteca Científica Brasileira, Série A II. Rio de Janeiro. 214p.

Nickel R., Schummer A. \& Seiferle E. 1981. The circulatory system, the skin, and the cutaneous organs of the domestic mammals, p.169-176. In: Ibid. (Eds), Anatomy of the Domestic Animals. Vol.3. Paul Parey, Berlin,

Oliveira M.F., Carter A.M., Bonatelli M., Ambrosio C.E. \& Miglino M.A. 2006. Placentation in the rock cavy (Kerodon rupestris Wied, 1820). Placenta 27:87-97.

Oliveira M.F., Miglino M.A., Ambrósio C.E., Assis Neto A.C., Moura C.E.B., Albuquerque J.F.G., Verechia F.T. \& Carvalho M.A.M. 2003. Funículo umbilical de fetos de mocós, Kerodon rupestris Wied, 1820. Revta Bras. Reprod. Anim. 27(2):290-292.

Peduti Neto J. \& Borelli V. 1970. Origem das artérias celíaca e mesentérica cranial, por tronco comum, em Felis catus domestica. Revta Fac. Med. Vet., São Paulo, 8:395-398.

Schmidt D.P. \& Schoenau L.S.F. 2007. Origem das artérias celíaca e mesentérica cranial por tronco comum em cão. Ciência Rural 37(2):408-411.

Silva F.O.C., Pereira C.C.H., Severino R.S., Santos A.L.Q., Drummond S.S., Bombonato P.P. \& Silva R.M. 1996. Comportamento das artérias mesentéricas cranial e caudal em macacos (Cebus apella). Anais 17을 Congresso Brasileiro de Anatomia, Fortaleza, p.191.

Souza W.M., Souza N.T.M., Miglino M.A., Machado M.F., Ferraz R.H.S. \& Duarte J.M.B. 1996. Ramificação das artérias mesentéricas cranial e caudal do veado catingueiro (Mazama govazoubira). Anais 17º Congresso Brasileiro de Anatomia, Fortaleza, p.191.

Thomaz J.M., Carvalho A.F., Miglino M.A., Maçanares C.A.F., Ambrosio C.E. \& Oliveira M.F. 2006. Caracterização morfológica dos dentes de mocó Kerodon rupestris (Mammalia: Rodentia). Braz. J. Vet. Res. Anim. Sci. 43:702-707.

Zogno M.A., Miglino M.A. \& Oliveira M.F. 2004. Análise bioquímica dos líquidos fetais e citologia do fluido amniótico da fêmea de mocó (Kerodon rupestris). Braz. J. Vet. Res. Anim. Sci. 41(4):226-235. 